\title{
Patient-reported outcomes following plantar incisions in foot surgery
}

\author{
Alwich N Alexander, ${ }^{1 *}$ (Dikiforos P Saragas, ${ }^{2}$ Paulo NF Ferrao ${ }^{2}$ \\ ${ }^{1}$ Linksfield Foot and Ankle Fellowship Programme; Department of Orthopaedics, University of Pretoria, Steve Biko Academic Hospital, Pretoria, \\ South Africa \\ ${ }^{2}$ Linksfield Foot and Ankle Fellowship Programme; Department of Orthopaedics, University of the Witwatersrand, Johannesburg, South Africa \\ ${ }^{*}$ Corresponding author: alwich23@gmail.com
}

Citation: Alexander AN, Saragas NP, Ferrao PNF. Patient-reported outcomes following plantar incisions in foot surgery. SA Orthop J 2021;20(4):208-212. http://dx.doi.org/10.17159/23098309/2021/v20n4a3

Editor: Dr Graham McCollum, University of Cape Town, Cape Town, South Africa

Received: October 2020

Accepted: January 2020

Published: November 2021

Copyright: @ 2021 Alexander AN. This is an open-access article distributed under the terms of the Creative Commons Attribution Licence, which permits unrestricted use, distribution and reproduction in any medium, provided the original author and source are credited.

Funding: No funding was received for this study.

Conflict of interest: The authors declare they have no conflicts of interest that are directly or indirectly related to the research.

\begin{abstract}
Background

Plantar incisions may be used in a variety of surgical procedures. Despite numerous studies reporting on procedures which use plantar incisions and thus inadvertently demonstrating good results with plantar incisions, most surgeons still avoid this approach due to the fear of developing a painful plantar scar. There is a shortage of studies demonstrating a clear correlation between plantar scar formation and poor patient-reported outcomes. The aim of this study is to assess the clinical outcome of plantar incisions in various procedures.
\end{abstract}

\section{Methods}

In this retrospective study we identified all patients who underwent surgery using a plantar incision between January 2000 and December 2019. A total of 23 patients were available for assessment. Three common procedures were identified: lesser metatarsal head resection, plantar fibromatosis excision and lateral sesamoidectomy. Demographic data was collected, and clinical outcome was assessed using the Self-Reported Foot and Ankle Score (SEFAS) questionnaire. Twenty-one female (22 feet) and two male patients (two feet) were included. The mean follow-up was 124 (range 8-231) months in the plantar fibromatosis group, 111.5 (range 28-177) months in the lateral sesamoidectomy group and 106.3 (range 42-157) months in the lesser metatarsal head excision group. The study included 12 patients in the sesamoidectomy, nine patients in the plantar fibromatosis and two patients in the lesser metatarsal head excision groups. The mean age of the study population was 45 (range 20-71) years.

\section{Results}

The mean postoperative SEFAS score in our series was 44 (range 22-48). Nineteen (82\%) patients scored as excellent, two (10\%) patients as good, one (4\%) patient as fair and one $(4 \%)$ as poor. All wounds healed well with no symptomatic callosities on clinical examination requiring revision.

\section{Conclusion}

This study demonstrates that plantar incisions, irrespective of indication and orientation (21 longitudinal and three transverse), heal well and with good patient-reported outcomes. We believe that it would be erroneous to 'avoid plantar incisions at all costs' and that plantar incisions must be considered if deemed technically superior and with less risk than a dorsal approach.

Level of evidence: Level 4

Keywords: plantar incisions, patient-reported outcome

\section{Introduction}

The plantar skin is unique in that it is considerably thicker than the rest of the foot. ${ }^{1}$ It is more keratinised, hairless, and filled with an abundance of sweat glands. ${ }^{1}$ Fibrous septae originate from the plantar fascia and attach to the plantar skin to provide increased mechanical support in resisting shear forces during the gait cycle. ${ }^{2}$ The superficial fascia has abundant fibrous-fatty tissue that assists in impact absorption. ${ }^{2}$ Courtiss et al. demonstrated that dermal collagen is orientated perpendicular to the axis of muscle and that the collagen in a scar is parallel to the direction of the scar. ${ }^{3}$ Hypertrophic scars can be caused by distraction or tension across an incision area. Since skin tension is greatest in the direction of the relaxed skin tension lines (RSTLs), an incision made parallel to the RSTLs would lessen the chance of painful hypertrophic scar formation. ${ }^{3,4}$ Also, the incision is then parallel to collagen bundles and perpendicular to muscle contraction, which results in finer and stronger scar lines., Unfortunately, most pathologies require a longitudinal incision which is perpendicular to the transversely oriented RSTLs (Figure 1).

Plantar incisions can be utilised in several pathologies, with the excision of Morton's neuromas being the most common indication. Plantar fibromatosis (Ledderhose disease) is a relatively rare pathology of the plantar aponeurosis characterised by disordered 

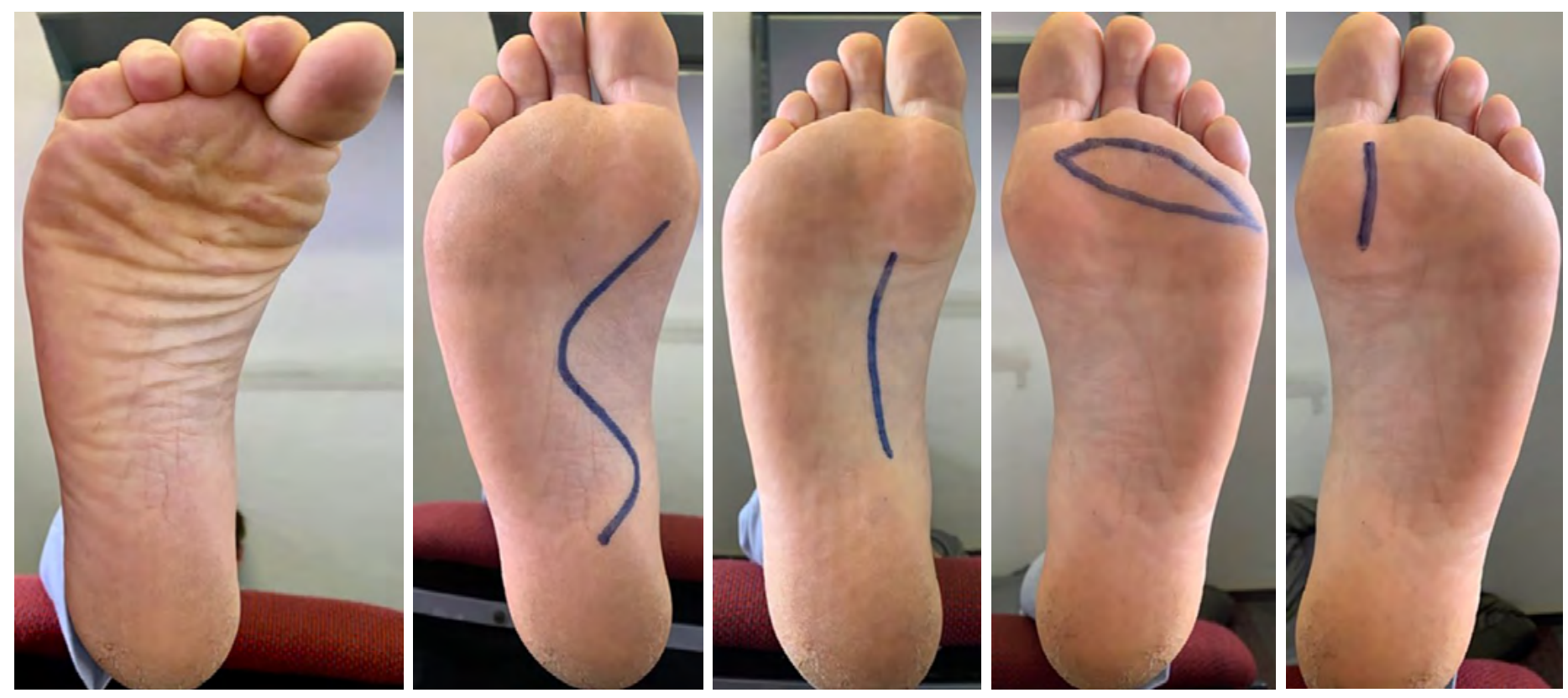

Figure 1. RSTL and surgical approaches

fibrous tissue proliferation and the subsequent formation of nodules. ${ }^{5}$ Surgical management has generally been reserved for pain relief, as this condition is of a benign nature. However, more recently, indications for surgery include both pain refractory to conservative treatment as well as local aggressiveness of the lesion. ${ }^{6}$ Three main techniques have been employed in the surgical management of plantar fibromatosis: local excision, wide excision and complete fasciectomy. Apart from recurrence, other reported complications that adversely affect patient outcomes include impaired wound healing, painful scarring, nerve entrapment and loss of arch height. ${ }^{6}$

Lateral (fibular) sesamoidectomy can be performed via a dorsal or plantar approach. The dorsal approach has been the preferred approach as it avoids the possibility of causing wound problems or the formation of irritating scars secondary to the plantar approach. ${ }^{7,8}$ However, excision of the lateral sesamoid via a dorsal approach can be difficult with suboptimal exposure and the risk of damage to the adductor mechanism and interdigital nerve.

Metatarsal head resection arthroplasty, as described by Hoffman, is a surgical technique in which a transverse plantar approach is utilised to excise the metatarsal heads. ${ }^{9}$ This procedure is described for complex forefoot deformities usually associated with irreducible dislocation of the metatarsophalangeal joints of the lesser toes. The technique has been modified by various surgeons with good outcomes. However, numerous complications, such as painful callosities associated with these procedures, have been reported. The extent to which these complications affect patient satisfaction, however, remains unclear.

Historically, the use of plantar surgical incisions by orthopaedic and foot surgeons alike have been avoided due to fears of complications, particularly scar formation in those patients for whom postoperative non-weight-bearing wound protection would be difficult. The use of this surgical approach was first published in 1940, with Betts et al. reporting its use for the resection of Morton's neuroma, yielding no complications. ${ }^{10}$ Despite numerous studies advocating plantar incisions for certain pathological entities, many surgeons still advise avoiding this approach if possible. We believe that plantar incisions heal well with little risk of a long-term symptomatic hypertrophic scar and do not necessarily correlate with poor subjective outcomes. The aim of this study was to evaluate patient-reported outcomes following plantar incisions and assess clinically for the development of hypertrophic and/or painful scar formation.

\section{Materials and methods}

Following ethical approval, we retrospectively reviewed the records of all patients who underwent foot surgery using a plantar incision between January 2000 and December 2019. Inclusion criteria included patients older than 18 years, and a minimum of six-months follow-up. Patients were excluded if their records were incomplete, or they were not available for follow-up. All surgeries were performed at a single centre by two foot-and-ankle surgeons. Subjective outcome was assessed using the validated Self-Reported Foot and Ankle Score (SEFAS) questionnaire. ${ }^{11}$ The SEFAS score ranges from 0 to 48 , with 0 representing the most severe disability and 48 as normal function. The grading is assessed as excellent when scoring > 41; good 34-41; fair 27-33; and poor $<27$. For statistical analyses, simple descriptive statistical tools were used. All numerical values are presented as a mean with a range. Basic demographic data were also recorded.

Twenty-three patients ( 24 feet) met the inclusion criteria and were available for clinical evaluation and to complete the questionnaire. The study population included 21 females and two males with a mean age of 45 (range 20-71) years and a mean follow-up of 116

Table I: Indications for lateral sesamoidectomy

\begin{tabular}{lc} 
Diagnosis & Number of patients \\
\hline Sesamoiditis & 8 \\
\hline Fracture & 2 \\
\hline Avascular necrosis & 1 \\
\hline Rheumatoid arthritis & 1 \\
\hline
\end{tabular}

Table II: Plantar fibroma size

\begin{tabular}{rccccccccc} 
Patient & P1 & P2 & P3 & P4 & P5 & P6 & P7 & P9 & P \\
Size in $\mathrm{mm}$ & $25 \times 15 \times 10$ & $30 \times 20 \times 8$ & $54 \times 13 \times 5$ & $38 \times 18 \times 10$ & $40 \times 20 \times 4$ & $20 \times 10 \times 5$ & $46 \times 10 \times 3$ & $17 \times 13$ & $30 \times 18 \times 5$ \\
\hline
\end{tabular}



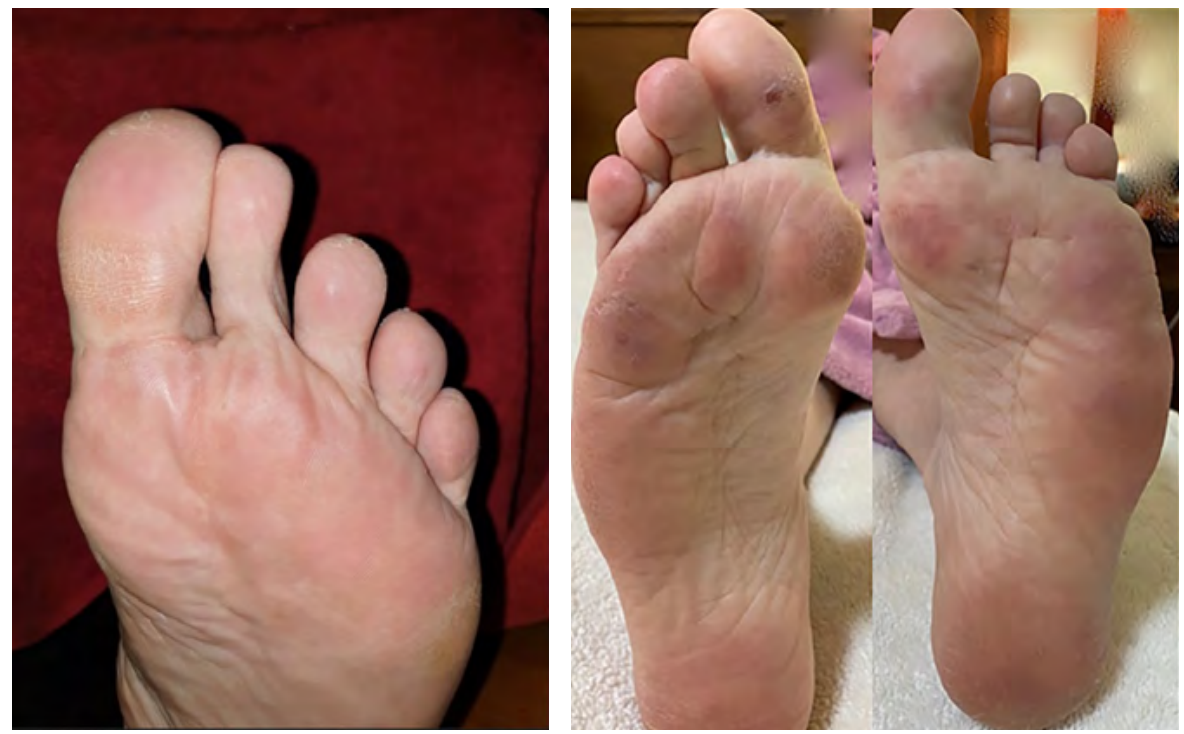

Figure 2. Healed scar following lateral sesamoidectomy
Figure 3. Healed scars following bilateral forefoot reconstruction through plantar elliptical incision

reconstruction, a plantar-based transverse incision, based over the lesser metatarsal heads, excising an elliptical piece of the overlying skin and superficial subcutaneous tissue was performed. Following careful soft-tissue dissection and protection of important structures, excision of the lesser metatarsal heads was carried out with an oscillating saw while maintaining the normal cascade of the lesser metatarsals. K-wires were then used to align the proximal phalanges to the metatarsals. The plantar incision was closed in a routine manner using 4-0 interrupted Nylon sutures while performing a dermodesis. The postoperative protocol entailed two weeks of strict elevation with weight bearing as tolerated in a forefoot offloading postoperative wedge shoe. Sutures were removed at three weeks and K-wires at six weeks post surgery.

\section{Results}

Five patients underwent surgical excision of plantar fibromatosis nodules through a lazy-S incision and four patients through a longitudinal

(range 8-231) months. Three common procedures were identified: lateral sesamoidectomy, excision of plantar fibromatosis and metatarsal head resection. The cohorts included 12 patients with lateral sesamoidectomy, nine patients with plantar fibromatosis excision and two patients with metatarsal head resections. The indications for lateral sesamoidectomy are outlined in Table $I$. The indications in the plantar fibromatosis group included eight solitary lesions and one patient with multifocal disease. All plantar fibromatosis patients presented with pain refractory to conservative management with no history of previous local steroid injections. All metatarsal head resections were performed as part of rheumatoid forefoot reconstruction. The mean follow-up was 111 (range 28177 ) months in the lateral sesamoidectomy group, 124 (range $8-231$ ) months in the plantar fibromatosis group, and 106 (range 42-157) months in the metatarsal head excision group.

\section{Operative technique}

In all conditions, surgery was performed with a thigh tourniquet under a bloodless field.

Sesamoidectomy: The lateral sesamoid was approached through a plantar curvilinear incision in the first web plantar crease. Soft tissue dissection was meticulous, taking care to preserve the plantar nerve and protect the flexor hallucis longus tendon and adductor mechanism. Following excision, the flexor hallucis brevis (FHB) was repaired. The wound was closed in layers and the skin closed with 4-0 Nylon interrupted sutures. The postoperative protocol entailed two weeks of strict elevation with weight bearing as tolerated in a forefoot offloading shoe. The hallux and second toe were taped together for a total of eight weeks to protect the repaired FHB. Physiotherapy was commenced at six weeks with transition to normal shoes.

Excision of plantar fibromatosis: A lazy-S or medially based plantar longitudinal incision was utilised for exposure of the plantar aponeurosis. Excision of the nodules with a $1 \mathrm{~cm}$ border of normal tissue was performed. Wounds were closed in a routine manner with 4-0 Nylon interrupted sutures for the skin. The postoperative protocol entailed two weeks of back slab immobilisation and strict non-weight-bearing. Sutures were removed at three weeks. Once the wound had healed fully, the patient was advised to massage the wound with an oil rich in vitamin $\mathrm{E}$, and weight bearing was allowed as tolerated in supportive shoes.

Metatarsal head resections: As part of the rheumatoid forefoot plantar medial approach. No patient received radiotherapy postoperatively. All specimens were confirmed histologically, and no patient developed recurrence of nodules during follow-up. The nodular sizes are reported in Table II.

In the metatarsal head resection group, both patients were being treated with methotrexate and the patient with bilateral disease used additional disease-modifying antirheumatic drugs (DMARDs) and steroids. A biologics-free window was strictly adhered to in this patient prior to having surgery.

None of the patients in the cohorts had delayed wound healing or wound infection necessitating additional surgical intervention. On clinical examination at final follow-up, all plantar incisions had healed well with no painful callosities or hypersensitivity requiring scar revision (Figures 2 and 3). The mean postoperative SEFAS score in our series was 44 (range 22-48). Nineteen (82\%) patients scored as excellent, two (10\%) patients as good, one (4\%) patient as fair and one (4\%) as poor. The SEFAS score according to procedure is reported in Table III.

The one patient who scored poorly had bilateral rheumatoid forefoot reconstruction, and complained of persistent swelling of the foot and the permanent need for orthotics due to recurrence of the lesser toe deformities. This patient had no complaints related to the plantar scars.

\section{Discussion}

There is a paucity in the literature addressing the subjective outcomes of plantar incisions. Most reports on the outcome of plantar incisions are indirectly addressed in studies evaluating

Table III: SEFAS grading

\begin{tabular}{|c|c|c|c|c|}
\hline $\begin{array}{l}\text { 을 } \\
\text { 휴 }\end{array}$ & 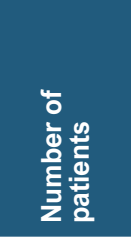 & 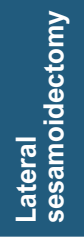 & 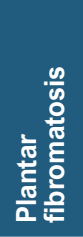 & 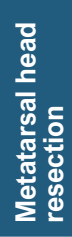 \\
\hline Excellent (> 41) & $19(82 \%)$ & 12 & 7 & \\
\hline Good (34-41) & $2(10 \%)$ & & 1 & 1 \\
\hline Fair (27-33) & $1(4 \%)$ & & 1 & \\
\hline Poor (<27) & $1(4 \%)$ & & & 1 \\
\hline
\end{tabular}


outcomes of various pathologies. ${ }^{7,9,10,12,13}$ There is still a reluctance to utilise this approach due to the fear of complications, particularly a painful scar.

To the best of our knowledge, Richardson et al. conducted the only study in modern literature, reviewing specifically the outcome of plantar incisions for a heterogenous group of conditions. ${ }^{14}$ They included 89 patients who underwent plantar incisions for conditions such as adductor release, Morton's neuroma excision and resection of metatarsal heads. Ninety-six per cent of these patients were satisfied with the outcome. The average follow-up in their study was 25 months, which is considerably less than the current study. In a prospective study assessing the outcome of longitudinal plantar incisions for excision of Morton's neuroma, Akermark et al. demonstrated that $90 \%$ and $98 \%$ of their study population reported none to slight scar tenderness and restriction of daily activity respectively. ${ }^{15}$

Ishie et al., Amin et al. and Hamalainen and Raunio all reported satisfactory results utilising a plantar approach for forefoot reconstruction in rheumatoid patients. ${ }^{16-18}$ However, none of these studies included a clear description regarding the effect of plantar scar formation on patient satisfaction. Matsumoto et al., and Hulse and Thomas demonstrated that painful callosity formation of the plantar scar is associated with recurrence of the deformity and is responsible for unsatisfactory outcomes in $40-58 \%$ of rheumatoid forefoot reconstruction cases. ${ }^{19,20}$ In comparison, Canedo et al. demonstrated a $100 \%$ patient satisfaction rate despite one patient developing a plantar callosity. ${ }^{21}$ It is important to be cognisant of the fact that rheumatoid arthritis is a chronic inflammatory disease which may lead to further foot involvement following forefoot reconstruction. This may lead to great variability in patient satisfaction as demonstrated by the above-mentioned results. In keeping with the literature, in our study both rheumatoid patients (one bilateral) reported well-healed scars with no hypersensitivity or pain, despite one patient scoring as poor (bilateral). This patient had recurrence of deformities requiring shoe modifications and orthotics, which was the reason for her dissatisfaction.

Plantar fibromatosis is a benign but locally aggressive fibrous tissue tumour. ${ }^{22}$ In a recent systematic review, the recurrence rate was reported to be $74.3 \%$ in primary cases; hence, most of the literature pertaining to this condition focuses on this complication. ${ }^{23}$ Sammarco and Mangone formulated a pre- and intraoperative tumour-staging system based on the nodularity, the presence of skin adherence, involvement of the plantar fascia and tumour extension to the overlying flexor tendon sheath (Table IV). ${ }^{24}$ This

\section{Table IV: Plantar fibromatosis tumour-staging system}

\begin{tabular}{cl}
$\begin{array}{c}\text { Tumour } \\
\text { grade }\end{array}$ & \multicolumn{1}{c}{ Description } \\
I & $\begin{array}{l}\text { Focal disease isolated to a small area on the medial and/or } \\
\text { central aspect of the fascia } \\
\text { No adherence to skin } \\
\text { No deep extension to the flexor sheath }\end{array}$ \\
\hline II $\quad \begin{array}{l}\text { Multifocal disease, with or without proximal or distal } \\
\text { extension } \\
\text { No adherence to the skin } \\
\text { No deep extension to the flexor sheath }\end{array}$ \\
$\begin{array}{l}\text { Multifocal disease, with or without proximal or distal } \\
\text { extension } \\
\text { Either adherence to the skin or deep extension to the flexor } \\
\text { sheath }\end{array}$ \\
$\begin{array}{l}\text { Multifocal disease, with or without proximal or distal } \\
\text { extension } \\
\text { Adherence to the skin and deep extension to the flexor } \\
\text { sheath }\end{array}$
\end{tabular}

tumour staging showed good correlation with postoperative wound complications and recurrence. Isolated and solitary nodules can be excised using a transverse approach not crossing the RSTLs. In this study, the majority of patients had extensive lesions (large or multinodular) which required a longitudinal incision crossing the RSTLs, in order to gain adequate exposure. Eight of the nine patients scored good to excellent on the SEFAS score with no wound complications or painful scar formation. Only one patient scored as fair and upon reviewing the patient's surgical records, it was noted that this patient had multifocal disease with tumour adherence to overlying skin (stage III).

Lateral sesamoidectomy can be performed through either a dorsal or plantar approach. The dorsal is the favoured approach as it avoids the risk of plantar wound complications but is technically more difficult unless there is a relatively large web space due to metatarsus primus varus. 7,8 Richardson et al. included 105 longitudinal plantar incisions for various conditions and although sesamoid conditions were not included, the plantar incisions used were not dissimilar to the plantar incision we used for lateral sesamoidectomy. ${ }^{14}$ They found six patients with punctate keratosis which was asymptomatic in two of the patients. The remaining four patients complained of a tender scar, necessitating altering their shoe wear in three of the four patients. Only two of these patients ultimately required excision of the keratosis and went on to heal without any sequelae. Other complications in this group included four patients with mild, intermittent tenderness as well as four wounds that dehisced. All these patients required no further interventions. All the 12 lateral sesamoidectomy patients in our study healed without complications. Saxena and Krisdakumtorn evaluated return to sport following fibular sesamoidectomy and reported that the three patients who underwent plantar incisions returned to impact activity two weeks earlier than the group in which a dorsal approach was used. ${ }^{25}$

The SEFAS is a validated patient-reported outcome measure (PROM), specifically for forefoot and ankle/hindfoot conditions. ${ }^{11}$ In the only other study utilising a scoring system to assess the surgical outcomes of plantar fibromatosis, Sammarco and Mangone reported an average postoperative AOFAS score of 77 and Maryland Foot Score of $86 .{ }^{24}$ These are functional scoring systems and do not account for the patient's satisfaction. This is the first study to report on outcomes using a validated patient-reported outcome score. The mean overall SEFAS score in our study was 44 , with $92 \%$ of patients scoring their outcomes as excellent or good. The only poor outcome was in the rheumatoid patient who had bilateral surgery as discussed above. We found no difference in the SEFAS scoring according to surgery-specific cohorts.

The limitations of this study include the small number of patients, limited procedures and retrospective design. In the literature, the most common indication for plantar incisions is excision of interdigital neuromas but in our unit, we routinely use dorsal incisions for both primary and revision cases.

\section{Conclusion}

Our findings show that plantar incisions heal well with a high patient satisfaction. We believe that good clinical outcome is directly related to sound surgical technique and meticulous soft tissue handling. Plantar incisions must be considered if they are deemed technically superior and with less risk of collateral damage as compared to using a dorsal approach, without the unfounded fear of developing a 'painful scar'.

\section{Ethics statement}

The authors declare that this submission is in accordance with the principles laid down by the Responsible Publication Position Statements as developed at the 2nd World Conference on Research Integrity in Singapore, 2010. 
Prior to the commencement of this study, ethical approval was obtained from the following ethical review board: Human Research Ethics Committee, HREC REF: M191168. Informed consent was obtained from all patients prior to being included in the study.

\section{Declaration}

The authors declare authorship of this article and that they have followed sound scientific research practice. This research is original and does not transgress plagiarism policies.

\section{Author contributions}

ANA: Study conceptualisation, data collection, data analysis, manuscript preparation, manuscript revision

NPS: Study conceptualisation, manuscript revision

PNFF: Study conceptualisation, manuscript revision

ORCID

Alexander AN (1) https://orcid.org/0000-0002-4023-7550

Saragas NP https://orcid.org/0000-0002-5566-7588

Ferrao PNF https://orcid.org/0000-0003-4639-0326

\section{References}

1. Thoolen M, Ryan TJ, Bristow I. A study of the skin of the sole of the foot using high frequency ultrasonography and histology. Foot. 2000;10:14-17.

2. Crowe CS, Cho DY, Kneib CJ, et al. Strategies for reconstruction of the plantar surface of the foot: A systematic review of the literature. Plast Reconstr Surg. 2019;143(4):1223-44.

3. Courtiss EH, Longacre JJ, Destefano GA, et al. The placement of elective skin incisions Plast Reconstr Surg. 1963;31(1):31-44.

4. Borges AF, Alexander JE. Relaxed skin tension lines, Z-plasties on scars, and fusiform excision of lesions. Br J Plast Surg. 1962;15:242-54.

5. Young JR, Sternbach S, Willinger M, et al. The etiology, evaluation, and management of plantar fibromatosis. Orthop Res Rev. 2019;11:1.

6. Carroll P, Henshaw RM, Garwood C, et al. Plantar fibromatosis: pathophysiology, surgical and non-surgical therapies: an evidence-based review. Foot Ankle Spec. 2018;11(2):168-76.

7. Milia MJ, Cohen BE, Anderson RB. Plantar approach for isolated fibular hallux sesamoidectomy. Tech Foot Ankle Surg. 2003;2:268-71.

8. Taylor CF, Butler M, Parsons SW. Problems associated with the excision of the hallux sesamoids. Foot Ankle Clin. 2014;19:425-36.

9. Hoffman P. An operation for severe grades of contracted or clawed toes. Am J Orthop. 1911;9:441.

10. Betts LO. Morton's metatarsalgia: neuritis of the fourth digital nerve. Med J Aust. 1940;1:514-15.

11. Coster MC, Rosengren BE, Bremander A, et al. Comparison of the Self-Reported Foot and Ankle Score (SEFAS) and the American Orthopedic Foot and Ankle Society Score (AOFAS). Foot Ankle Int. 2014;35(10):1031-36.

12. Kates A, Kessel L, Kay A. Arthroplasty of the forefoot. J Bone Joint Surg. 1967;49B:552-57.

13. Fowler AW. A method of forefoot reconstruction. J Bone Joint Surg Br. 1959;41:507-13.

14. Richardson EG, Brotzman SB, Graves SC. The plantar incision for procedures involving the forefoot. JBJS. 1193;75(5):726-31.

15. Akermark C, Saartok T, Zuber Z. A prospective 2-year follow-up study of plantar incisions in the treatment of primary intermetatarsal neuromas (Morton's neuroma). J Foot Ankle Surg. 2008;14(2):67-73

16. Ishie S, Ito H, Azukizawa M, et al. Delayed wound healing after forefoot surgery in patients with rheumatoid arthritis. Mod Rheumatol. 2015;25(3):367-72.

17. Amin A, Cullen N, Singh D. Rheumatoid forefoot reconstruction. Acta Orthop Belg 2010;76(3):289-97.

18. Hamalainen M, Raunio P. Long term followup of rheumatoid forefoot surgery. Clin Orthop Relat Res. 1997;340:34-8.

19. Matsumoto $T$, Kadono $Y$, Nishino J, et al. Midterm results of resection arthroplasty for forefoot deformities in patients with rheumatoid arthritis and the risk factors associated with patient dissatisfaction. J Foot Ankle Surg. 2014:53(1):41-6.

20. Hulse N, Thomas AM. Metatarsal head resection in the rheumatoid foot: 5-year follow-up with and without resection of the first metatarsal head. J Foot Ankle Surg 2006:45(2):107-12.

21. Do Couto Canedo L, Pereira Filho MV, et al. Effect of plantar incision for metatarsa head resection arthroplasty of the small toes. Scientific Journal of the Foot \& Ankle. 2018;12(2):117-22.

22. Kadir HKA, Chandrasekar CR. Partial fasciectomy is a useful treatment option for symptomatic plantar fibromatosis. Foot. 2017;31:31-34.

23. Fuiano M, Mosca M, Caravelli S, et al. Current concepts about treatment options of plantar fibromatosis: A systematic review of the literature. J Foot Ankle Surg. 2019;25:559-64.

24. Sammarco GJ, Mangone PG. Classification and treatment of plantar fibromatosis. Foot Ankle Int. 2000;21:563-9.

25. Saxena A, Krisdakumtorn T. Return to activity after sesamoidectomy in athletically active individuals. Foot Ankle Int. 2003;24:415-9. 